\title{
The Precise Methods for the Measurement of Collimator Hole Angulation and Center of Rotation of SPECT by Adaptive Quality Control Phantom
}

\author{
Hossein Zamani Zeinali ${ }^{*}$, Mehran Ataee ${ }^{2}$, Hamid Ravanbakhsh ${ }^{3}$, Ehsan Masumi Goodarzi ${ }^{3}$, \\ Samana Ghoreishi ${ }^{4}$, Gholamreza Raisali1 \\ ${ }^{1}$ Application of Radiation Research School, Nuclear Science and Technology Research Institute, Atomic Energy \\ Organization of Iran (AEOI), Karaj, Iran \\ ${ }^{2}$ Nuclear Science and Technology Research Institute (AEOI), Tehran, Iran \\ ${ }^{3}$ Department of Physics, Peyambare Azam Institute, Islamic Azad University of Tehran, Tehran, Iran \\ ${ }^{4}$ Parto Control in Advance Co., Tehran, Iran \\ Email: "hzeinali@nrcam.org
}

Received 26 June 2014; revised 3 August 2014; accepted 19 August 2014

Copyright @ 2014 by authors and Scientific Research Publishing Inc.

This work is licensed under the Creative Commons Attribution International License (CC BY).

http://creativecommons.org/licenses/by/4.0/

(c) () Open Access

\section{Abstract}

The Adaptive Quality Control Phantom (AQCP) is a computer-controlled phantom which positions and moves a radioactive source in the Field of View (FOV) of an imaging nuclear medicine device on a definite path to produce a spatial distribution of gamma rays to perform $Q C$ Tests such as the Collimator Hole Angulation (CHA) and the Center of Rotation (COR) of Single Photon Emission Computer Tomography (SPECT). The collimator hole angulation for six collimators was measured using a point source and a computer-controlled cylindrical positioning system. In this method, the displacement of the image of a point source was examined as the AQCP was moving point source vertically away from the collimator face. The results of the high-accuracy measurement method of CHA show that the measurement accuracy for absolute angulation errors is better than $\pm 0.024^{\circ}$. The Root Mean Square (RMS) of CHA for LEHR, LEHS and LEUHR collimators of SMV dual heads camera and LEGP, MEGP and HEGP of GE Millennium MG were evaluated to be $0.290^{\circ}, 0.292^{\circ}$, $0.208^{\circ}, 0.154^{\circ},{0.220^{\circ}}^{\circ}$ and $0.202^{\circ}$, respectively. It is to be added in this connection that the evaluated RMS of CHA for LEHR collimator with the distance variation from the collimator's surface $\pm 1 \mathrm{~mm}$ has been varied \pm 0.04 degree. A new method for the center of rotation assessment by AQCP is introduced and the results of this proposed method as compared with the routine QC test and their differences are discussed in detail. We defined and measured a new parameter called Dynamic Mechanical Error (DME) for applying the gantry motion correction.

\footnotetext{
"Corresponding author.
}

How to cite this paper: Zeinali, H.Z., Ataee, M., Ravanbakhsh, H., Goodarzi, E.M., Ghoreishi, S. and Raisali, G. (2014) The Precise Methods for the Measurement of Collimator Hole Angulation and Center of Rotation of SPECT by Adaptive Quality Control Phantom. World Journal of Nuclear Science and Technology, 4, 208-215.

http://dx.doi.org/10.4236/wjnst.2014.44026 


\title{
Keywords
}

\author{
Nuclear Medicine, Adaptive Quality Control Phantom, Collimator Hole Angulation, Center of \\ Rotation
}

\section{Introduction}

Single Photon Emission Computed Tomography (SPECT) has a number of advantages relative to conventional Nuclear Medicine (NM) imaging including Contrast improvement and Total volume imaging [1]-[6]. In order to realize these advantages, rigorous QC procedures must be performed on a routine basis. Important considerations for tomography, unlike planar imaging, include flood field uniformity and Center of Rotation (COR) correction/verification and Collimator Hole Angulation (CHA) [1] [4] [7] [8]. For SPECT imaging, the angulation of the holes in parallel hole collimator must be known in order to ensure its proper set up during a tomographic acquisition, i.e., its holes must be perpendicular to the Axis of Rotation [2] [4] [6]. There are different methods for evaluating hole angulation [1]-[3] [6] [7]. But the one which is a quantitative method and was used by the authors is the displacement of the image of a point source examined as the source is moved vertically away from the collimator face. To apply such method, it is necessary to use a phantom like Adaptive Quality Control Phantom (AQCP) which holds the source at two vertical distances from the collimator face to ensure the accurate alignment of the source [2] [4]. We tried to measure CHA for three low energy collimators with more accuracy by AQCP method.

In reconstructing the images from projections during a SPECT acquisition, it is assumed that image matrix, representing the activity distribution in a section, has a constant relationship to the data acquisition matrix [2] [4] [5] [8]. If one matrix shifts to the other for different angles of data acquisition, then the image reconstructed from back projecting the data will be blurred because of the relative motion of the two matrixes [2] [8]. The elements contributing to COR consist of mechanical problems with detector rotation, changes in amplifier gain and offset, problem with head tilt, collimator hole angulation, errors in the analog to digital converter, as well as the lack of parallelism between the collimator/detector plane and the axis of rotation [4]. In the IAEA-TECDOC602 method of COR evaluation, the constant shift of COR correction was measured and applied [8]. We tried to obtain dynamic mechanical rotation error of gamma camera SPECT by evaluating the results of COR with IAEA-TECDOC-602 and AQCP methods.

AQCP is designed to perform a uniform set of procedures that can be used for routine quality control of a scintillation camera-based system. AQCP is an electromechanical device designed to acquire the field uniformity, center of rotation and collimator hole angulation by using some advanced methods of position control [9][12]. In order to use AQCP to simulate QC phantoms, the device moves a radioactive source within the Field Of View (FOV) of the NM imaging device on a definite path to produce a three-dimensional artificial distribution of $\gamma$ rays [11] [13] [14]. AQCP, in fact, can be used to optimize QC tests for the measurement of uniformity, resolution, linearity, absolute size of pixel and center of rotation and can perform the total performance test of Single Photon Emission Computer Tomography (SPECT) [11] [12] [14].

\section{Materials and Methods}

\subsection{AQCP Design and Structure}

AQCP consist of different mechanical and electrical parts and is equipped with hardware and software systems. Cylindrical coordinates are used as a pattern for the motion and simulation of AQCP [9] [11]-[13]. A combination of ball screw and reel system is used to position the point source in any desired point in space with a precision of $0.06 \mathrm{~mm}$ (Figure 1). Two step motors are used; each of them is connected to a gear box and divides each complete turn into 2000 steps (0.18 degree/step). The accuracy of step motor is $10 \%$ ( 0.018 degree/step). To achieve the coordinates $Z$ and $\theta$ by the AQCP software, the ball screw system is used (Figure 1 ).

Ball screw consists of a precise screw with a step of $1 \mathrm{~mm}$ and a wide nut (4 cm full length) (Figure $1(\mathrm{c})$ ). Another small nut was put under the wide nut. The $\theta$ parameter is adjusted by changing the position of the small nut and fixing it to the wide nut. When the small nut is open, the system can give different $Z$ 's. So, in this system 


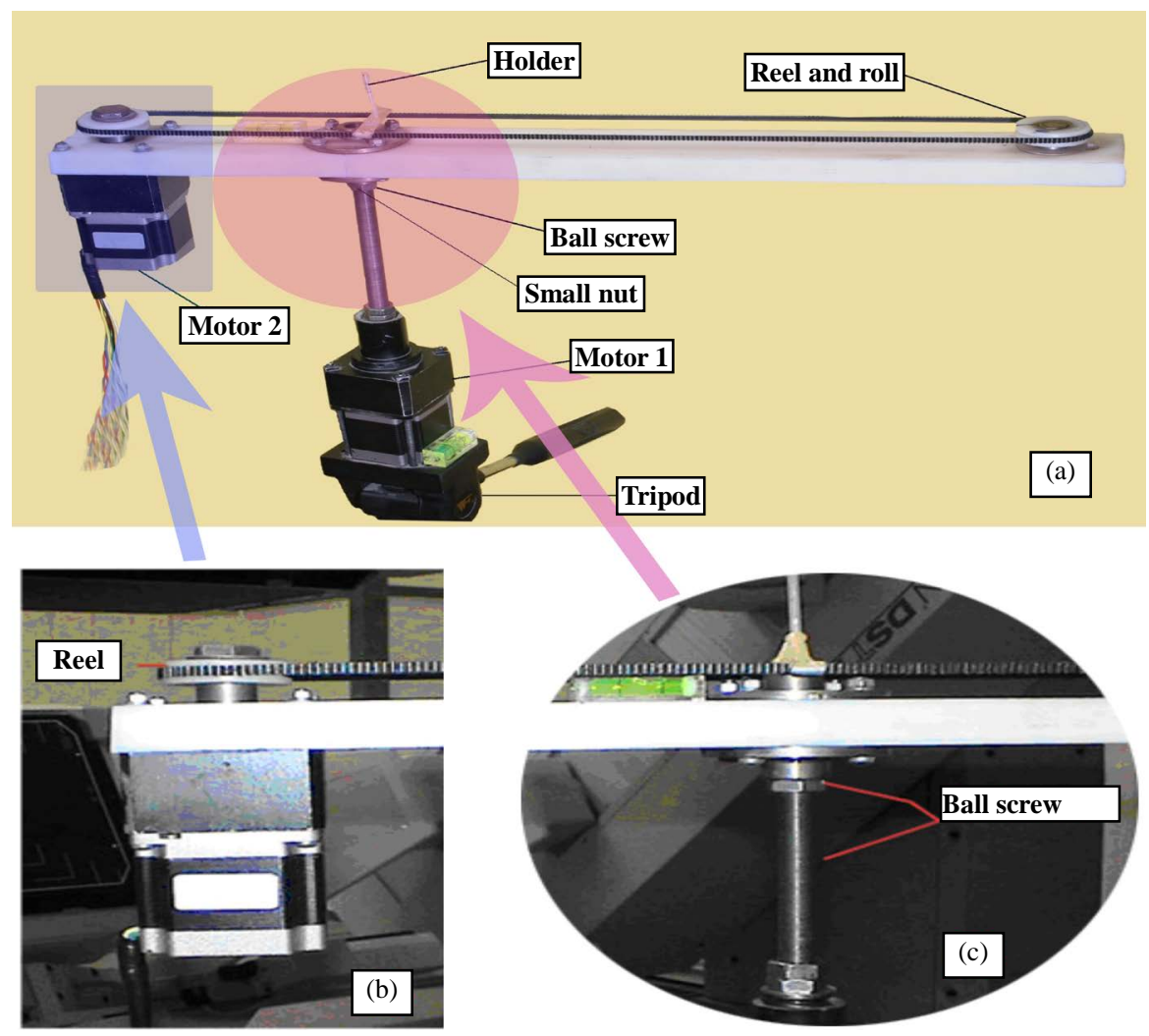

Figure 1. (a) AQCP mechanical section. Ball screw and reel and roll systems are used to position the point source in any desired point in space with a precision of $0.06 \mathrm{~mm}$. (b) To get the related $r$, motor rotates the reel and the roll. (c) To get the $Z$ and $\theta$ the ball screw system is used. Ball screw consists of a precise screw with a step of $1 \mathrm{~mm}$ and with a wide nut ( $4 \mathrm{~cm}$ length). Another small nut is under the wide nut. The $\theta$ parameter is adjusted by changing the position of the small nut and fixing it to the wide nut. When the small nut is open, the system can give different $Z$. So, in this system the $Z$ and $\theta$ can be adjusted manually.

$Z$ and $\theta$ coordinates can be adjusted manually (Figure 1(c)) and generally small nut is closed when the AQCP is operating. To reach $r$ (third dimension of the cylindrical coordinates), the related motor (motor No. 2 In Figure 1(b)) rotates the reel and the roll. A complete round of the reel $(30 \mathrm{~mm})$ is 500 steps, as a result; the precision of transverse radius of cylindrical coordinates $(r)$ is $0.06 \mathrm{~mm}$ (Figure 1(b)). From electrical point of view, AQCP is equipped with two driver and actuator circuits of step motors which together with other parts help the whole system function and operate properly and accurately. The overall functions of AQCP are controlled by a software interface developed to position the cylindrical coordinates [9]-[14]. The user of the system would be able to define and design a path for moving the radioactive source. The software draws the path on the screen and analyzes the results of the experiment.

The Haematocrit-capillaries with external diameter of 1.2 - $1.3 \mathrm{~mm}$ and a radionuclide, i.e. ${ }^{99 \mathrm{~m}}$ Tc with high specific activity (more than $50 \mathrm{mCi} / \mathrm{cc}$ ) were used to make a point. A small drop of ${ }^{99 \mathrm{~m}} \mathrm{Tc},{ }^{67} \mathrm{Ga},{ }^{201} \mathrm{Tl}$ or ${ }^{131} \mathrm{I}$ with a diameter of $1 \mathrm{~mm}$ was used to make the point source. The gamma camera SPECT systems under examination were SMV double head gamma camera model DSX-XL SPECT and GE Millennium MG.

\subsection{Collimator Hole Angulation (CHA) Test}

In order to measure the CHA by AQCP, the point sources were positioned in 140 points within the FOV under the following defined conditions: length of the source $\leq 2 \mathrm{~mm}$, Inner diameter of the source $\leq 1.2 \mathrm{~mm}, 200 \mu \mathrm{Ci}$ activity of ${ }^{99 \mathrm{~m}} \mathrm{Tc},{ }^{67} \mathrm{Ga},{ }^{201} \mathrm{Tl}$ or ${ }^{131} \mathrm{I}$, window: $20 \%$. The CHA was evaluated for the camera fitted with 6 collimators including Low Energy High Resolution (LEHR), Low Energy High Sensitivity (LEHS) and the Low 
Energy Ultra High Resolution (LEUHR) of SMV camera and Low Energy General Purpose (LEGP), Medium Energy General Purpose (MEGP) and High Energy General Purpose (HEGP) collimators of GE Millennium MG. The characteristics of collimators are shown in Table 1.

For each of those collimators listed above, two images were taken at 140 different points of collimators with matrix size of $256 \times 256$ at two vertical distances of $Z_{1}=10 \mathrm{~cm}$ as position 1 and $Z_{2}=13 \mathrm{~cm}$ as position 2 from the collimator and at each point of the Prepare Your Paper before Styling collimators positions, the point source was imaged for a total of $50 k$ counts.

The data analysis of the two images as described in position 1 and 2 is initiated applying nine point smoothing kernel to reduce the random fluctuation in the data. The approximate $X$ and $Y$ coordinates (COGX and COGY) of each point source image are determined by calculating the centroids of each point source image from relations 1 and 2 [4] [5]. COGX and COGY are, in fact, the estimated locations of point source image for each 140 points in image matrix.

$$
\begin{aligned}
& \operatorname{COGX}=\frac{\sum i \times C(i, j)}{\sum C(i, j)} \\
& \operatorname{COGX}=\frac{\sum j \times C(i, j)}{\sum C(i, j)}
\end{aligned}
$$

Here, $C(i, j)$ is count in pixel $(i, j)$. For each of the 140 points of the collimators the CHAX and CHAY were determined by relations 3 and 4 [2] [4] [5].

$$
\begin{aligned}
\operatorname{CHAX} & =\operatorname{Tan}^{-1}\left(K_{1} \frac{\left[\operatorname{COGX}\left(Z_{1}\right)-\operatorname{COGX}\left(Z_{2}\right)\right]}{\left(Z_{1}-Z_{2}\right)}\right) \\
\operatorname{CHAY} & =\operatorname{Tan}^{-1}\left(K_{1} \frac{\left[\operatorname{COGY}\left(Z_{1}\right)-\operatorname{COGY}\left(Z_{2}\right)\right]}{\left(Z_{1}-Z_{2}\right)}\right)
\end{aligned}
$$

In relation 3 and 4: $K_{1}=$ Pixel size $(\mathrm{mm} / \mathrm{pixel})$ and $\left(Z_{1}-Z_{2}\right)$ distance between two images in $\mathrm{mm}$ (for all measurements $30 \pm 0.06 \mathrm{~mm}$ ). The Root Mean Square (RMS) of CHA can be obtained by:

$$
\mathrm{CHA}=\sqrt{\mathrm{CHAX}^{2}+\mathrm{CHAY}^{2}}
$$

\subsection{Center of Rotation Test}

According to the IAEA-TECDOC-602 method for assessment of COR, the radioactive point source is placed at a distance $\mathrm{R}$ off the axis of rotation and a SPECT acquisition through $360^{\circ}$ around this point source is obtained. For COR measurement, it acquired a 360 degree tomographic study using a $64 \times 64$ matrix, 20\% window and 64 projections. Each projection requires $50 \mathrm{~K}$ counts acquisition [4] [5] [8]. The specifications of the point source are as follows: length of the source $\leq 2 \mathrm{~mm}$, Inner diameter of the source $\leq 1.6 \mathrm{~mm}$ and $200 \mu \mathrm{Ci}$ activity of ${ }^{99 \mathrm{~m}} \mathrm{Tc}$.

Table 1. Summary of collimator characteristics.

\begin{tabular}{cccccc}
\hline Label & Length $(\mathrm{mm})$ & Hole $(\mathrm{mm})$ & Septa $(\mathrm{mm})$ & Sensitivity $(\mathrm{Cpm} / \mu \mathrm{Ci})$ & System Resolution at $10 \mathrm{~cm}$ \\
\hline LEHR & 46 & 2.032 & 0.152 & 235 & $8.0 \mathrm{~mm}$ \\
LEUHR & 47 & 1.778 & 0.127 & 176 & $7.1 \mathrm{~mm}$ \\
LEHS & 47 & 2.870 & 0.203 & 460 & $10.7 \mathrm{~mm}$ \\
LEGP & 43 & 2.5 & 0.25 & - & 10.2 \\
MEGP & 42 & 3.0 & 1.2 & - & 12.7 \\
HEGP & 48 & 3.4 & 1.65 & - & 13.8 \\
\hline
\end{tabular}


The COGX and the COGY were calculated in each projection using relations 1 and 2. Offset error $R(\theta)$ as a function of rotation angle $(\theta)$ was calculated by relation 6 [4]. To further explain $R(\theta)$, in fact, it indicates the difference between the estimated and the measured point source image in each projection in $X$ axis. The constancy of the $y$-axis alignment during rotation should be calculated after a 90 degree rotation of the raw data. The COGY offset should be independent of angle so that almost a linear plot should be obtained [4] [8].

$$
R(\theta)=\frac{[(N+1)-\operatorname{COGX}(\theta)-\operatorname{COGX}(\theta+180)]}{2}
$$

Here, $N$ (matrix size $)=64$.

Considering the specific characteristics of AQCP, a new method has been introduced for COR assessment. In this new method the camera is held in a fixed position and AQCP moves the point source on the circle with radius $R$. In other words, AQCP rotates and positions the point source on the center of rotation of AQCP (cor) with the radius R (Figure 2). For the assessment of COR in the AQCP method, axis of AQCP rotation should be in parallel with the axis of SPECT rotation. Offset error $R_{-}$cor $(\theta)$ of AQCP method is calculated pursuing the same relation 6 as used in the IAEA-TECDOC-602 method.

In comparing the test results of the two afore-described methods, the Dynamic Mechanical Error (DME) of gantry in each angle was calculated by relation 7 .

$$
\sin (\Delta \theta)=\frac{\left[R(\theta)-R_{-} \operatorname{cor}(\theta)\right]}{R}
$$

In relation 7, $\Delta \theta$ is the Dynamic Mechanical Error of gantry which serves as a function of SPECT rotation angle $(\theta)$.

\section{Results}

\subsection{Collimator Hole Angulation (CHA) Test}

In the CHA measurement, the results of the three collimators of LEHR, LEHS and LEUHR of SMV camera and

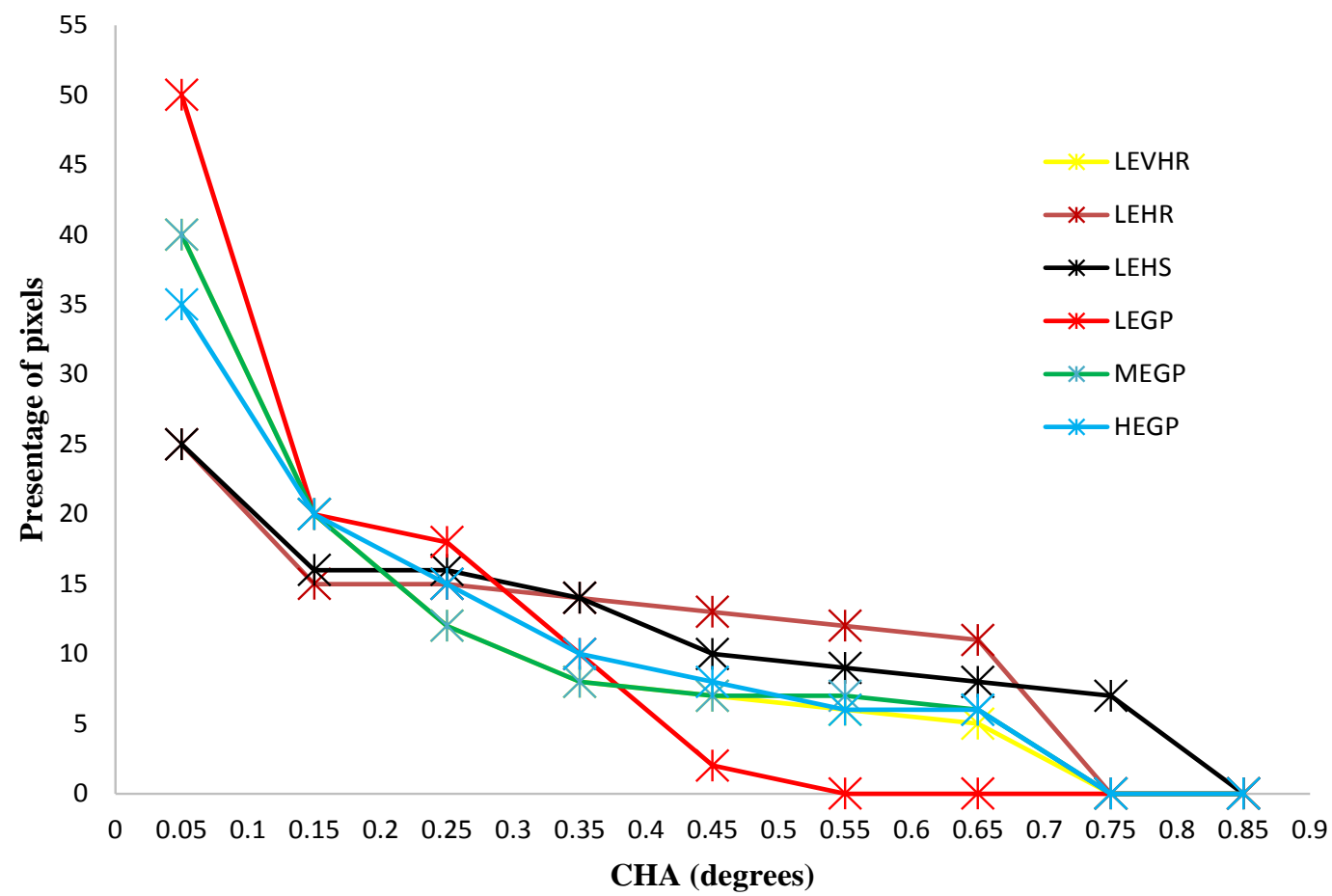

Figure 2. Statistical changes in CHA for LEHR, LEHS and LEUHR collimators of SMV camera and LEGP, MEGP and HEGP of GE Millennium MG. 
LEGP, MEGP and HEGP of GE Millennium MG were examined and the results obtained are shown in Figure 2 and Table 2. The images for LEHR CHA assessment were shown in Figure 3.

Repeated measurements for the three types of collimators including those obtained after re-running of the AQCP program gave an S.D. less than $0.1^{\circ}$ for each point of collimator. CHA test was conducted within an average time of 30 - $35 \mathrm{~min}$. The RMS ( \pm S.D.) of CHA for 140 positions of LEHR, LEHS and LEUHR collimators of SMV camera were $0.290^{\circ}\left( \pm 0.207^{\circ}\right), 0.292^{\circ}\left( \pm 0.197^{\circ}\right)$ and $0.208^{\circ}\left( \pm 0.184^{\circ}\right)$ respectively. CHA test was conducted within an average time of 30 - $35 \mathrm{~min}$. The RMS ( \pm S.D.) of CHA for 140 positions of LEGP, MEGP and HEGP collimators of GE Millennium MG camera were $0.158^{\circ}\left( \pm 0.120^{\circ}\right), 0.220^{\circ}\left( \pm 0.201^{\circ}\right)$ and $0.292^{\circ}$ $\left( \pm 0.211^{\circ}\right)$ respectively.

The Maximum of CHA for LEHR, LEHS and LEUHR collimators of SMV camera were $0.78^{\circ}, 0.67^{\circ}$ and $0.66^{\circ}$ respectively. The Maximum of CHA for LEGP, MEGP and HEGP collimators of Millennium MG camera were $0.56^{\circ}, 0.63^{\circ}$ and $0.64^{\circ}$ respectively.

In addition, it has been proved that the evaluated RMS of CHA for LEHR collimator with the distance variation from the collimator's surface of $\pm 1 \mathrm{~mm}$ has been varied only \pm 0.04 degree. The results for LEHR collimator indicate that a $1 \mathrm{~mm}$ change in $\left(Z_{1}-Z_{2}\right)\left(\left(Z_{1}-Z_{2}\right)=(31 \mathrm{~mm})\right)$ changes the RMS of CHA from $0.29^{\circ}$ to $0.33^{\circ}$ and $-1 \mathrm{~mm}$ change in $\left(Z_{1}-Z_{2}\right)\left(\left(Z_{1}-Z_{2}\right)=(29 \mathrm{~mm})\right)$ changes the RMS of CHA from $0.29^{\circ}$ to $0.25^{\circ}$.

It should be mentioned that CHA is dependent on the variation of the distance of the two images. For precise measurement of CHA, the precise distance of the point source movement should be measured vertically away from collimator face.

Table 2. CHA test results.

\begin{tabular}{cccc}
\hline Collimator & RMS of CHA (degree) & Standard Deviation (degree) & Maximum of CHA (degree) \\
\hline LEHR & 0.290 & 0.207 & 0.78 \\
LEHS & 0.292 & 0.197 & 0.67 \\
LEUHR & 0.209 & 0.184 & 0.66 \\
LEGP & 0.158 & 0.120 & 0.56 \\
MEGP & 0.220 & 0.201 & 0.63 \\
HEGP & 0.292 & 0.211 & 0.64 \\
\hline
\end{tabular}
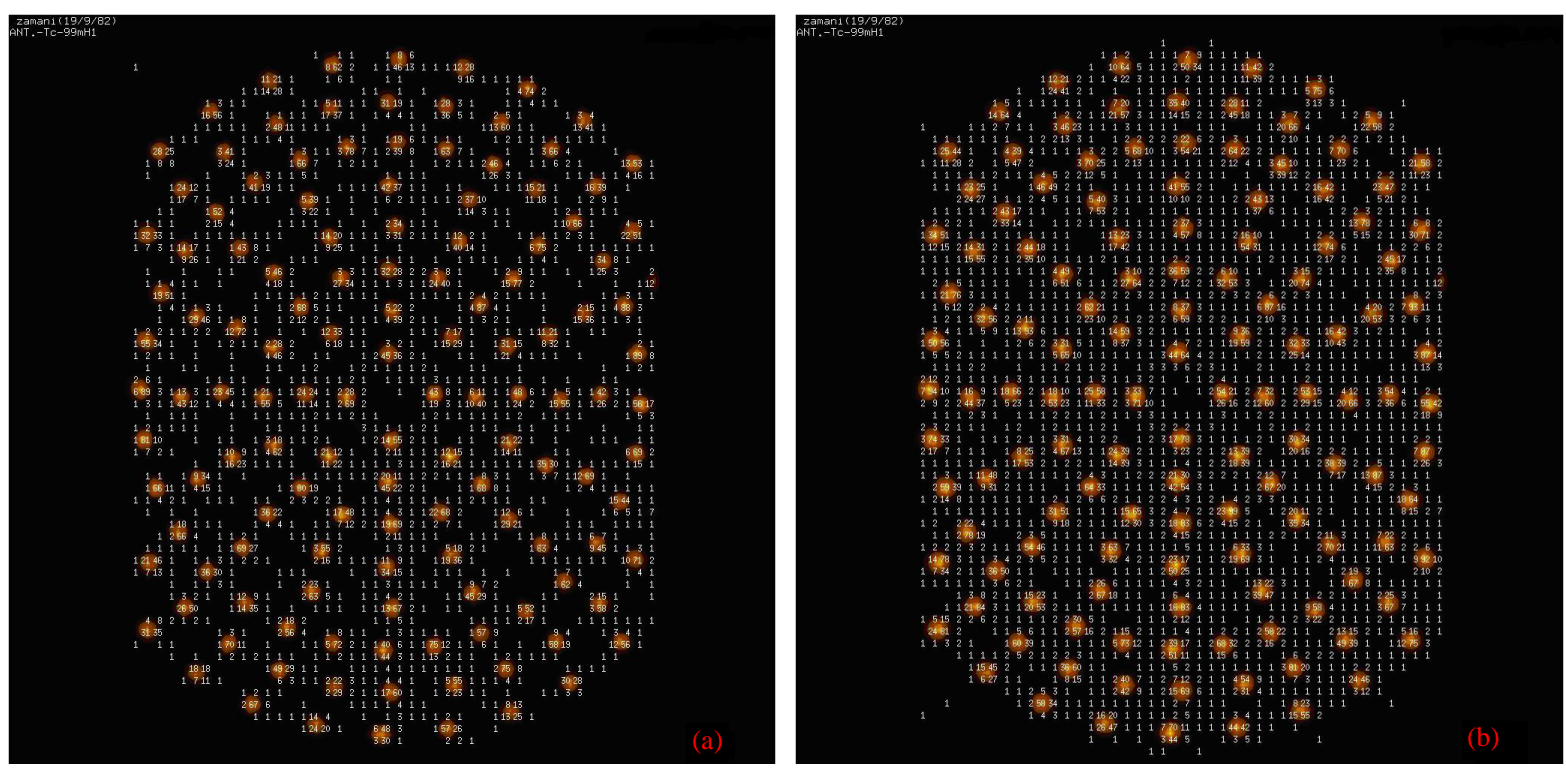

Figure 3. Two images were taken at 140 different points of LEHR collimator for measuring the CHA with AQCP method. The image $A$ is taken in $10 \mathrm{~cm}$ distance and the image $B$ in $13 \mathrm{~cm}$ distance from collimator surface. 


\subsection{Center of Rotation Test}

The results of measurement of COR offset $(R(\theta))$ in terms of SPECT rotation angle and the results of COR offset and error $\left(R \_\right.$cor $\left.(\theta)\right)$ in terms of AQCP rotation angle can be seen in Table 3. COR test was conducted within an average time of 8 - 10 min. with the two afore-said methods.

The calculation results of the Dynamic Mechanical Error (DME) and/or the mechanical problem/error with detector rotation in terms of SPECT rotation angle. The absolute mean ( \pm S.D.) of DME is $0.136( \pm 0.047)$ degrees.

\section{Discussion}

Collimator Hole Angulation (CHA) Test: These are very similar to the results obtained in other relevant studies [1] [2] [6]. Since AQCP can position the point source with absolute accuracy of $0.024^{\circ}$ therefore the CHA can be measured more precisely and interpreted visually. So it shows that the precise measurement of the distance of the two images $\left(\left(Z_{1}-Z_{2}\right)\right.$ in relations 3 and 4$)$ has a great effect on the measurement of CHA.

Center of Rotation test: The experiments show that there is a remarkable difference in the determination of COR offset and standard deviation between the IAEA-TECDOC-602 method and AQCP method. By comparing the findings of AQCP method with IAEA-TECDOC-602 method, it was found that the rotation of SPECT system is not ideally precise and accurate.

As shown in Table 3, the standard deviation of COR in $X$ and $Y$ Axis in the IAEA-TECDOC-602 Method are 0.28 and 0.31 whilst in AQCP method the standard deviation of COR in $X$ and $Y$ Axis are 0.269 and 0.293 . All of these measurements are conducted in Pixel Unit. These two parameters (COR offset and Standard Deviation) are varied due to mechanical problems with detector rotation, changes in amplifier gain and offset, problem with head tilt, collimator hole angulation, errors in the analog to digital converter, as well as the lack of parallelism between the collimator/detector plane and the axis of rotation [4] [5]. In IAEA-TECDOC-602 method of COR evaluation, COR was measured by applying a constant shift of COR correction and then it measured dynamic mechanical rotation error of gamma camera SPECT by evaluating the results of COR with IAEA-TECDOC-602 and AQCP methods.

Initial adjustment of axis of rotation of AQCP was very difficult because it had to be parallel to the axis of rotation of SPECT. The authors found here that because of the different shifts in the Y-axis of COR evaluated per different angles of SPECT rotation, the axis of rotation of AQCP was not parallel to the axis of rotation of the camera. Such maladjustment of the AQCP axis of rotation is considered as a source of error in our measurements but, in the meantime, it has a low impact on COR evaluation. The reason is that the authors succeeded to measure the COR with less offset error and standard deviation per angle of rotation with AQCP method compared to the IAEA-TECDOC-602 method. Based on such comparison between the two afore-described methods, it seems that the mechanical problems with detector rotation should be considered as the main cause of the difference between the two methods under consideration.

Due to the significance of the matter, the authors tried to introduce the motion correction of the detector rotation in comparison with the deviation of COR evaluated by the two above methods. Our COR test results show 7\% difference between the maximum deviations of COR in the two methods under discussion.

Consequently, the authors found out that by applying the correction of detector rotation in different angles of projection, which will be discussed in a separate paper, the image reconstructed from back projection of SPECT will not be blurred, and this would result in a higher resolution image.

\section{Conclusions}

AQCP, as a computerized and automated phantom with unique features in design and construction, is capable of

Table 3. The COR offset error of SPECT with IAEA-TECDOC-602 and AQCP methods (all measurement in pixel unit).

\begin{tabular}{ccccccc}
\hline Method & $\begin{array}{c}\text { Maximum and } \\
\text { minimum offset } \\
\text { of X axis }\end{array}$ & $\begin{array}{c}\text { Error rang in } \\
\text { X axis }\end{array}$ & $\begin{array}{c}\text { Deviation of } \\
\text { X offset }\end{array}$ & $\begin{array}{c}\text { Maximum and } \\
\text { minimum offset } \\
\text { of Y axis }\end{array}$ & $\begin{array}{c}\text { Error rang in } \\
\text { Y axis }\end{array}$ & $\begin{array}{c}\text { Deviation of } \\
\text { Y offset }\end{array}$ \\
\hline $\begin{array}{c}\text { IAEA-TECDOC-602 } \\
\text { method }\end{array}$ & $0.73,-0.49$ & 1.23 & 0.28 & $0.57,-0.69$ & 1.26 & 0.31 \\
AQCP method & $0.53,-0.41$ & 0.94 & 0.269 & $0.52,-0.61$ & 1.13 & 0.293 \\
\hline
\end{tabular}


being adapted for diverse and various QC test measurements. AQCP produces spatial distribution of gamma rays to simulate QC phantom for acceptance and/or routine QC testing.

AQCP has some advantages compared to any other similar QC measurement methods which briefly include the following: reduction of radioactive material consumption, reduction of radiation exposure to the staff, reduction of QC test cost, implementation of QC program with one phantom, performing a uniform set of procedures, increasing the accuracy and precision of some QC tests, and automation of the measurements and evaluation processes.

\section{Acknowledgements}

The authors of the present paper should present their special thanks and appreciation to the administration and the staff of Karaj Nuclear Medicine and Agricultural Research Center as well as Mr. Mehdi Zamani Zeinali for his cordial assistance.

\section{References}

[1] Malmin, R.E., Peggy, C.S. and Guth, W.R. (1990) Collimator Angulation Error and Its Effect on SPECT. Journal of Nuclear Medicine, 31, 655-659.

[2] Busemann-Sokole, E. (1986) Measurement of Collimator Hole Angulation and Camera Head Tilt for Slant and Parallel Hole Collimators Used in SPECT. Journal of Nuclear Medicine, 28, 1592-1598.

[3] Chang, W, Li, S.Q., Williams, J.J., et al. (1988) New Methods of Examining Gamma Camera Collimators. Journal of Nuclear Medicine, 29, 674-683.

[4] (1987) Rotating Scintillation Camera SPECT Acceptance Testing and Quality Control. AAPM Report No. 22, American Association of Physicists in Medicine, College Park.

[5] Quality Control of Nuclear Medicine Instruments (1991) IAEA TECDOC 602. International Atomic Energy Agency, Vienna.

[6] Eckholt, M. and Bergmann, H. (2000) Angulation Errors in Parallel-Hole and Fan Beam Collimators: Computer Controlled Quality Control and Acceptance Testing Procedure. Journal of Nuclear Medicine, 41, 548-555.

[7] Takahashi, Y., Murase, K., Higashno, H., et al. (2002) SPECT Imaging with Off-Set Detector System: Comparison of Sampling Angles 2, 4 and 6 Degrees. Annals of Nuclear Medicine, 16, 343-347. http://dx.doi.org/10.1007/BF02988623

[8] Hines, H., Kayayan, R., Colsher, J., et al. (1999) Recommendations for Implementing SPECT Instrumentation Quality Control. Nuclear Medicine Section-National Electrical Manufacturers Association (NEMA). European Journal of Nuclear Medicine and Molecular Imaging, 26, 527-532. http://dx.doi.org/10.1007/s002590050421

[9] Thomas, G.B. (1969) Calculus and Analytic Geometry. 4th Edition, Addison Wesley, Reading, 340-470.

[10] National Instruments Corporation. www.NI.com

[11] Zeinali, H.Z., Ghiassi-Nejad, M. and Mirzai, A. (2007) Design an Adaptive Quality Control Phantom to Optimize QC Test Methods. The Open Medical Imaging Journal, 1, 1-6. http://dx.doi.org/10.2174/1874347100701010001

[12] Zeinali, H.Z. and Mirzai, A. (2008) Design an Adaptive Quality Control Phantom to Optimize Integral and Differential Uniformity, Collimator Hole angulation and Center of Rotation of SPECT. The Current Medical Imaging Reviews, 4, 1-8. http://dx.doi.org/10.2174/157340508784356743

[13] Kuo, B.C. (1987) Automatic Control Systems. 5th Edition, Prentice-Hall, Upper Saddle River, 290-345.

[14] Zeinali, H.Z., Ghiassi-Nejad, M. and Mirzai, A. (2008) Design an Adaptive Quality Control Phantom to Optimize Uniformity, Collimator Hole Angulation and Center of Rotation of SPECT. The Journal of Instrumentation, 3, 1-8. http://dx.doi.org/10.1088/1748-0221/3/02/P02009 
Scientific Research Publishing (SCIRP) is one of the largest Open Access journal publishers. It is currently publishing more than 200 open access, online, peer-reviewed journals covering a wide range of academic disciplines. SCIRP serves the worldwide academic communities and contributes to the progress and application of science with its publication.

Other selected journals from SCIRP are listed as below. Submit your manuscript to us via either submit@scirp.org or Online Submission Portal.
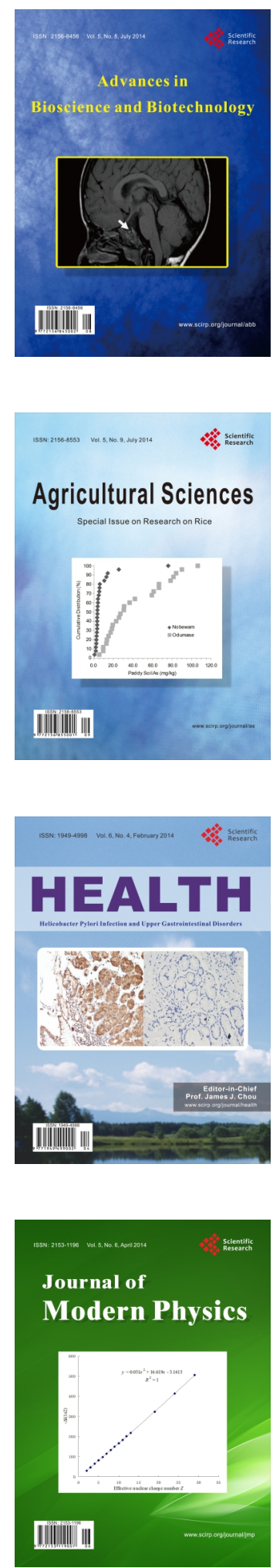
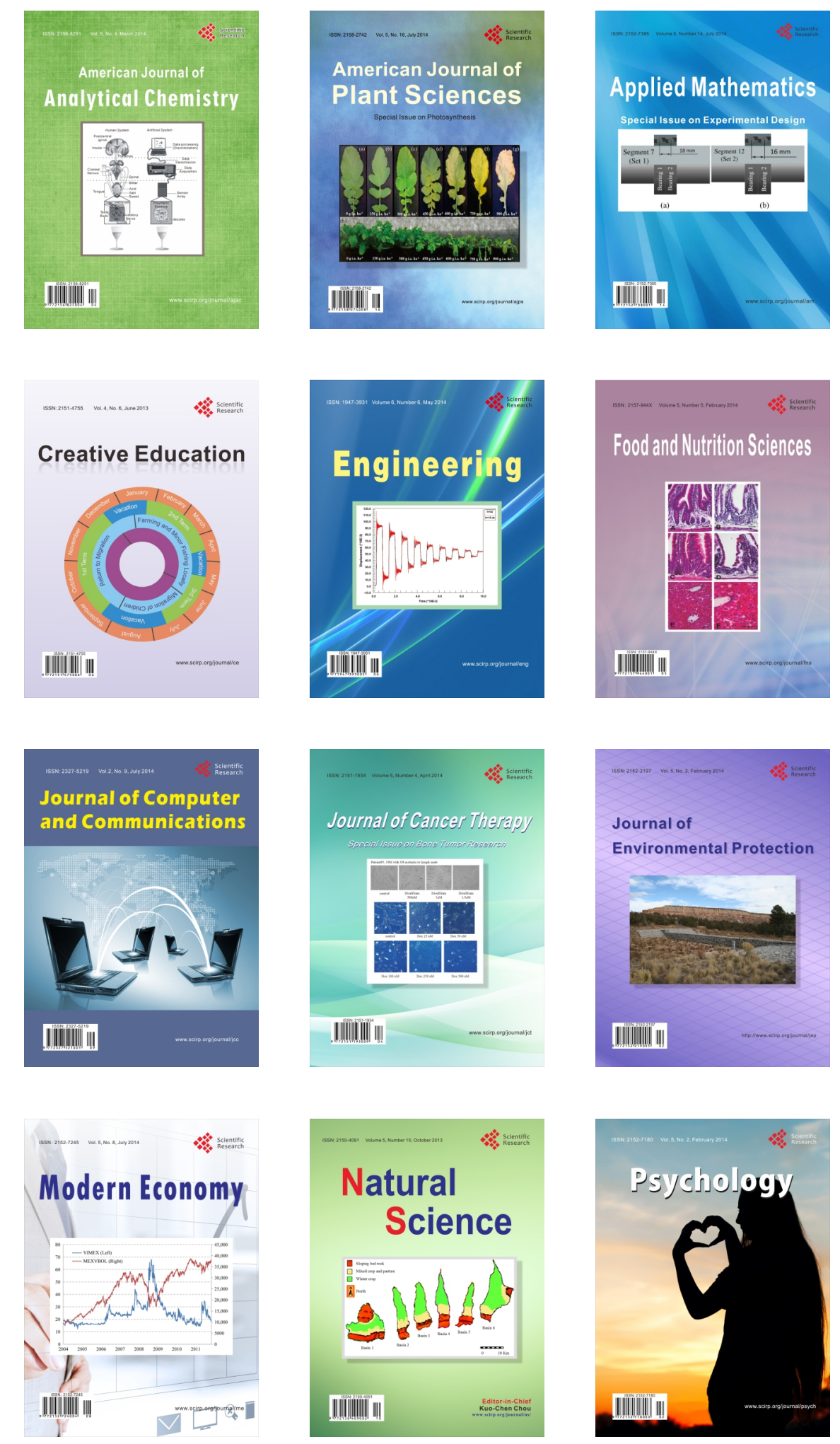